\title{
Diagnosis of Aortic Valve Stenosis Based on PCG Signal Using Wavelet Packet Decomposition (WPD) and Parametric Models
}

\author{
Pegah Derakhshan Mehr, Nader Jafarnia Dabanloo, Gholamreza Attarodi, Keivan Maghooli, Nazanin Hemmati \\ Islamic Azad University, Science and Research Branch ,department of Biomedical Engineering,Tehran,Iran
}

\begin{abstract}
Our aim in this study was to diagnose aortic valve stenosis from PCG signals using methods of converting the wavelet packet and statistical parameters. For categorization of three subcategories, K-nearest neighbor and multi-layer perceptron were used. After applying the proposed method on the PCG signals, the expected results were obtained. The highest classification accuracy for the features that were combined with the principal component analysis method and given to the nonlinear kernel classifier with a non-linear kernel of order 4 was $94.48 \%$ (without the principal component analysis method) and $98.38 \%$ (by the principal component analysis method). Therefore, the results of the classification show that our proposed method has been able to distinguish with the high ability of the phonocardiogram signals of a person with aortic valve stenosis and a healthy person. The accuracy of the multilayer perceptron and the nearest neighbor of $\mathrm{K}$ were also found to be $98 \%$ in detecting the stenosis of the aortic valve from the other. In fact, after reducing the number of features with PCA (composition of features), the classification accuracy of the diagnosis of aortic valve stenosis was increased by $5.13 \%$ and $2.96 \%$, respectively, using the KNN and MLP classifications. Also, after reducing the number of features with the PCA (feature combination), the SVM classifier with a nonlinear kernel was $2.91 \%$ and for the linear kernel increased by $15.42 \%$.
\end{abstract}

Keyword: Aortic valve stenosis, Phonocardiogram, wavelet packet decomposition

\section{Introduction}

Aortic valve stenosis is the most important disease of the heart valve in developed countries, which affects most people over the age of 65 . The normal function of the aortic valve is that the blood flows from the left ventricle to the systemic environment without any interruption or reversal. The natural aortic valve is completely closed and, when open, is a triangular aperture, which is smaller than the area of the crosssection itself. The valve stenosis occurs when the valve does not open completely. Early diagnosis of this disease can save lives, reduce the burden of the treatment of the disease, such as surgery. In addition, today, with increasing technological advances and the need for newer and less invasive technologies, the diagnosis of aortic stenosis using PCG signals is considered non-invasive, functional, and cheaper than other Methods have been considered and we will use this signal in this study. In the studies of this area, the use of the wavelet transformation is usually used [1-5]. But here we are going to use the wavelet packet decomposition method because the use of WPD can produce a better resolution for the decomposed signal. In this study We plan to diagnose this disease by analyzing the phonocardiogram (PCG) signal with a wavelength packet (WP) and extracting the parameters of the parameters model such as AR, ARMA. To do this, the first and second sounds of the heart are extracted from the PCG signals with the aid of a vowel pack. Then the coefficients of the parameterized models are calculated and given as the input of the classifier, the supporting vector machine (SVM), and the output of the problem is the diagnosis of the stenosis of the aortic valve or the health of the valve.

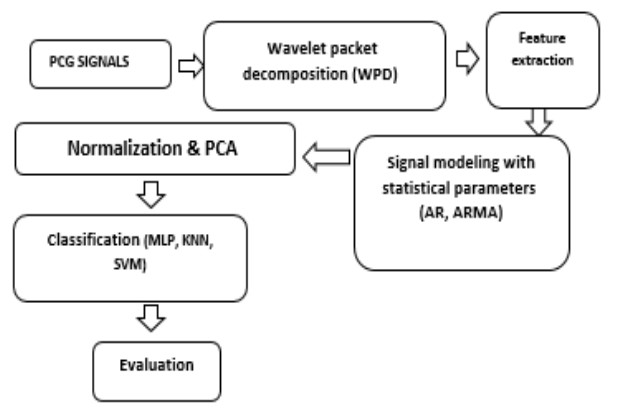

Figure1.Block diagram of the proposed system

\section{Concepts}

2-1 Phonocardiography :Phonocardiogram is a device for recording heart sounds and murmurs. This personal and individual interpretation system eliminates heart sounds, as well as evaluates heart 
and murmur sounds for electrical and mechanical events in the heart cycle[6].

2-2 .Wavelet packet decomposition: wavelet packet decomposition(WPD) is a wavelet that signal is converted into a signal in which the signal passes through more filters than the discrete wavelet transform. In the transformation of a discrete wavelet, in each step the coefficient of approximation is passed through the downstream and upstream filters, but in the transformation of the bundle of packets, both the approximation and partial coefficients of the filter pass.



Figure 2 - Decomposition tree a: Discrete wavelet Conversion b: wavelet packet Analysis [7]

The advantage of using the WP analysis is that it allows the combination of different levels of analysis to make the original signal possible.

2-3 .Feature extraction: In this dissertation, Shennot's entropy features, mean, standard deviation, power, Skwness and kurtosis were extracted from audio signals.

2-4 .Parameter models: Sometimes one can equate the signal with a parametric model and estimate the parameters of this model, which can be used as a feature in the classification. In this paper, the signal is considered as a linear combination of signal and white noise (in the past and present moments (models AR and ARMA) and the coefficients of the model as Features are considered.[8]

After normalizing all the features due to the high dimensionality dimension, we chose the selected properties using the feature selection methods.

\section{Method}

In this study, PCG signals related to the murmur database were used. This database is about Johns Hopkins Medical School. The data was taken from www.mrmurlab.com. The data includes 20 sonic aortic valve stenosis of $20 \mathrm{~s}$ and 23 normal sounds of $20 \mathrm{~s}$. The sampling frequency of the PCG signals is $44100 \mathrm{~Hz}$. According to the block diagram, implementation is done as follows: Extraction of features only from the wavelet packet transform coefficients for different base functions, Extract features only from statistical coefficients with different methods, Extract the characteristics of the wavelet packet decomposition coefficients and statistical coefficients, The parameter $k$ in the class of the KNN, Number of hidden layer neurons in the MLP class, Linear and nonlinear kernel in SVM class, The nonlinear kernel order in the SVM class. At all stages, $80 \%$ of the features were used for training and $20 \%$ equal to the test. The classification has been performed 100 times and the average results of the evaluation criteria have been reported.

3-1 .SVM classification results: In this section, all features, such as the wavelet packet transform the kernel and the statistical models, were assigned to the SVM class.

Table (1). results of SVM classification in different scenariosdB4

\begin{tabular}{|c|c|c|c|c|}
\hline classifier & sensivity & specifity & percision & accuracy \\
\hline $\begin{array}{c}\text { (SVM) linear( } \\
\text { kernel) }\end{array}$ & 89.15 & 86.03 & 76.20 & 81.23 \\
\hline $\begin{array}{c}\text { (SVM )non linear } \\
\text { kernel(3rd } \\
\text { order) }\end{array}$ & 91.87 & 89.64 & 83.32 & 85.45 \\
\hline $\begin{array}{c}\text { (SVM ) non } \\
\text { linear kernel(4rd } \\
\text { order) }\end{array}$ & 100 & 91.27 & 86.90 & 94.48 \\
\hline $\begin{array}{c}\text { (SVm)l non linear } \\
\text { kernel( 5rd } \\
\text { order) }\end{array}$ & 88.12 & 85.97 & 79.12 & 83.21 \\
\hline
\end{tabular}

\section{3-2 .Wavelet packet with various base functions}

According to Table 2, the classification accuracy was extracted for the features that were extracted from the wavelet transform with a Daubechies dB4(4order) base function, more than the other basic functions of the wavelet (Morelot, Mexican hat, HAR). This accuracy is $82.42 \%$.

Table(2). Wavelet packet with various base functions

\begin{tabular}{|c|c|c|c|c|c|}
\hline & $\begin{array}{l}\text { Mother } \\
\text { wavelet }\end{array}$ & $\begin{array}{c}\text { KNN } \\
(\mathrm{K}=5) \\
(\%)\end{array}$ & $\begin{array}{c}\text { MLP } \\
(N=5) \\
(\%)\end{array}$ & $\begin{array}{l}\text { SVM ) non } \\
\text { linear } \\
\text { kernel( } \\
\%)\end{array}$ & $\begin{array}{l}\text { SVM } \\
\text { linear } \\
\text { kernel } \\
(\%)\end{array}$ \\
\hline \multirow{4}{*}{$\begin{array}{c}2 \\
\text { Page } 2\end{array}$} & Haar & 82.29 & 77.12 & 80.95 & 74.15 \\
\hline & $\mathrm{dB}(4)$ & 79.43 & 74.50 & 82.42 & 75.01 \\
\hline & Mexican hat & 78.65 & 74.98 & 78.65 & 70.32 \\
\hline & Morlet & 79.13 & 76.45 & 78.65 & 71.43 \\
\hline
\end{tabular}


3-3 .Parametric models : In this section, only the features of the AR and ARMA parameter coefficients were given to the above-mentioned categories. The coefficients of the model were calculated using youlwalker (YW), BURG, forward-backward (FB), least squared (LS) methods. After extraction, the aforementioned features were calculated and normalization was done.

Table (3). feature extraction using coefficients of parametric models for different methods

\begin{tabular}{|c|c|c|c|c|}
\hline method & $\begin{array}{c}\text { KNN } \\
(\mathrm{K}=5) \\
(\%)\end{array}$ & $\begin{array}{c}\text { MLP } \\
(\mathrm{N}=5) \\
(\%)\end{array}$ & $\begin{array}{c}\text { SVM } \\
\text { (non } \\
\text { linear } \\
\text { kernel(\%) }\end{array}$ & $\begin{array}{c}\text { SVM } \\
\text { (linear( } \\
\text { kernel)(\%) }\end{array}$ \\
\hline YW & 83.29 & 79.23 & 78.54 & 75.86 \\
\hline FB & 75.98 & 76.32 & 73.54 & 72.11 \\
\hline BURG & 84.03 & 80.11 & 83.42 & 81.12 \\
\hline LS & 86.76 & 82.96 & 85.02 & 83.96 \\
\hline
\end{tabular}

3-4. The effect of the $k$ parameter on the class of KNN: In this section, features of the wavelet transform with dB4 and the statistical models are given for different values of $\mathrm{K}$ to the KNN class.

Table 4. shows the result of the KNN classifier for different

\begin{tabular}{|c|c|c|c|c|}
\hline classifier & $\begin{array}{c}\text { sensivity } \\
(\%)\end{array}$ & $\begin{array}{c}\text { specifity } \\
(\%)\end{array}$ & $\begin{array}{c}\text { percision } \\
(\%)\end{array}$ & $\begin{array}{c}\text { accuracy } \\
(\%)\end{array}$ \\
\hline $\begin{array}{c}\text { KNN } \\
(k=3)\end{array}$ & 89.98 & 81.57 & 76.10 & 84.18 \\
\hline $\begin{array}{c}\text { KNN } \\
(k=5)\end{array}$ & 96.43 & 91.28 & 87.23 & 93.25 \\
\hline $\begin{array}{c}\text { KNN } \\
(k=7)\end{array}$ & 88.90 & 85.34 & 82.49 & 86.43 \\
\hline
\end{tabular}

According to the above table, for the $K=5$ value, the best accuracy of the classification of the two categories of aortic valve stenosis and the lack of tightness is $93.25 \%$.

\section{3-5.The effect of the number of hidden neurons in the MLP classifier}

In this section, the features of the wavelet transform with dB4 (due to superiority to other basic functions of the violin) and the statistical models for several numbers of neurons in the latent layer are given to the MLP classification.

Table 5. shows the result of the MLP classifier for a number of hidden neurons

\begin{tabular}{|c|c|c|c|c|}
\hline Classifier & $\begin{array}{c}\text { Sensivity } \\
(\%)\end{array}$ & $\begin{array}{c}\text { Specifity } \\
(\%)\end{array}$ & $\begin{array}{c}\text { percision } \\
(\%)\end{array}$ & $\begin{array}{c}\text { Accuracy } \\
(\%)\end{array}$ \\
\hline $\begin{array}{c}\text { MLP } \\
(n=3)\end{array}$ & 75.23 & 71.36 & 74.12 & 74.5 \\
\hline $\begin{array}{c}\text { MLP } \\
(n=4)\end{array}$ & 89.76 & 85.12 & 81.34 & 83.24 \\
\hline $\begin{array}{c}\text { MLP } \\
(n=5)\end{array}$ & 99.17 & 91.25 & 86.93 & 94.16 \\
\hline
\end{tabular}

3-6 .Using the composition method of the principal component analysis component: According to the pervious table, the best accuracy is obtained for 5 neurons in the hidden layer. Thus, for the diagnosis of aortic valve stenosis, the highest average accuracy is $94.16 \%$.So far, the results of the classification have been distinguished in such a way that we separate the features manually (wavelet and parametric models), now we want to use the method of reducing the

Table (6) -Results of the classifier after the reduction of features with PCA

\begin{tabular}{|c|c|c|c|c|}
\hline $\begin{array}{c}\text { classifie } \\
r\end{array}$ & $\begin{array}{c}\text { sensivity } \\
\text { (\%) }\end{array}$ & $\begin{array}{c}\text { specifity } \\
(\%)\end{array}$ & $\begin{array}{c}\text { percision } \\
(\%)\end{array}$ & $\begin{array}{c}\text { accuracy } \\
\text { (\%) }\end{array}$ \\
\hline $\begin{array}{l}\text { MLP } \\
(n=5)\end{array}$ & 99.87 & 92.54 & 90.39 & 97.12 \\
\hline $\begin{array}{l}\mathrm{KNN} \\
(\mathrm{K}=5)\end{array}$ & 99.34 & 99.42 & 92.67 & 98.38 \\
\hline $\begin{array}{l}\text { SVM } \\
\text { (linear } \\
\text { kernel) }\end{array}$ & 96.43 & 97.82 & 91.14 & 96.65 \\
\hline $\begin{array}{l}\text { SVM } \\
\text { ( non } \\
\text { linear } \\
\text { kernel) }\end{array}$ & 97.64 & 95.12 & 92.21 & 97.39 \\
\hline
\end{tabular}

number of features (composition of the characteristic) of the main component analysis. To do this, we give all the characteristics to the PCA after normalization, and we give 10 combinations of superior features to the three classes of MLP, SVM, and KNN classes. Select floor segment parameters according to the best results $(\mathrm{N}=5$ and $\mathrm{K}=5)$. The results of this step are shown in Table 6.

According to this table, after reducing the number of features with PCA (composition of features), classification accuracy of the diagnosis of aortic valve stenosis increased by $5.13 \%$ and $2.96 \%$, respectively, using the KNN and MLP classifications. The classification accuracy for the SVM classifier with the nonlinear kernel was $2.91 \%$ and for the linear kernel increased by $15.42 \%$.

4 .Conclusion: In this study, the purpose of diagnosis of aortic valve stenosis was based on the signal obtained from the phonocardiogram. The tool used in this study was to transform the wavelet packet that analyzes the signals in both high and low frequency bands. Different basic functions such as Hawk, Mexican hat, Daubechies (4th order) and so on were used to analyze the signals of the phonocardiogram. In addition to this method, parametric models are also used: AR and ARMA. Different methods such as Burg, Yull Walker and ... were used to derive model 
parameters. Each of these features (violet packet and parametric models) was given to three commonly used backup carriers, $\mathrm{k}$ nearest neighbor and multilayer perceptron, and the classification of the two groups of aortic valve stenosis and so on. In each of these categories, their parameters were set up as trial and error, and the classification was done. In the classifier, the vector of support for linear and nonlinear kernels was used. In the class of KNN, we changed the value of the parameter $K$, and in the multi-layer perceptron, we changed the number of hidden layer neurons. Then, the criteria for sensivity, specifity,accuracy, percision and accuracy were calculated for each floor class. In addition, ROC charts were drawn. The number below the ROC curve (ROC area) represents the ability of the classifier to be segregated. In summary, the following results are obtained: The accuracy of KNN and SVM classifications was better than MLP, After reducing the number of features with PCA (composition of features), the classification accuracy of the diagnosis of aortic valve stenosis increased by $5.13 \%$ and $2.96 \%$, respectively, using the KNN and MLP classifications. After reducing the number of features with PCA (composition of features), the classification accuracy of the aortic valve stenosis diagnosis problem was increased by $2.91 \%$ for the non-linear kernel for the SVM class and for the linear kernel by $15.42 \%$, The highest accuracy of the SVM classification is obtained for the nonlinear kernel and rank 4 (94.48\%), The number of nodes in the secret layers of the MLP classifier with a try and error of 5 was obtained. This value has the best accuracy (94.16\%), The $\mathrm{K}$ number of neighbors in the KNN with a try and error of 5 is obtained. This value has the best accuracy (93.25\%), The extracted features of a 4-dB dB4 bulletin board have the highest accuracy for the SVM classification (nonlinear kernel), compared with other baseline base functions (82.42\%), The characteristics of the AR and ARMA statistical models with LS (least squares) method have the highest accuracy for the KNN class (86.76\%),According to these results, we can say that using our proposed method, we were able to obtain the label of stenosis of the aortic valve without stenosis of $94.48 \%$ (without PCA) and $98.38 \%$ (with PCA) on the corresponding PCG signals. Bring us This figure indicates that our proposed method has been able to demonstrate our goal with our high ability.
5 .Suggestions: To increase the ability of our proposed method, we have the following suggestions: You can use the" $s$ "conversion to decomposition the phonocardiogram signals, Nonlinear features can be used such as sample entropy, chaotic features such as correlation dimension, Lyapunov faces, or frequency features. To achieve the results of other studies, it is possible to use feature selection methods such as principal component analysis, or to use evolutionary methods to select optimal properties such as genetic algorithm, particle swarm and ..., Data exploration methods can also be used.

\section{References}

[1] El-Asir, B., Khadra, L., Al-Abbasi, A. H., \& Mohammed, M. M. J. Multi-resolution analysis of heart sounds. In Electronics, Circuits, and Systems, 1996. ICECS'96., Proceedings of the Third IEEE International Conference on (Vol. 2, pp. 1084-1087).

[2] Nayad Kouras, Daoud BOUTANA, Messaoud BENIDIR. Wavelet based segmentation and time-frequency characterization of some abnormal heart sound signals, 2012, 24th International Conference on Microelectronics (ICM).

[3] Voss, A., Herold, J., Schroeder, R., Nasticzky, F., Mix, A., Ulrich, P., \& Huebner, T. (2003, September). Diagnosis of aortic valve stenosis by correlation analysis of wavelet filtered heart sounds. In Engineering in Medicine and Biology Society, 2003. Proceedings of the 25th Annual International Conference of the IEEE (Vol. 3, pp. 2873-2876).

[4] Zin, Z. M., Salleh, S. H., Daliman, S., \& Sulaiman, M. D. Analysis of heart sounds based on continuous wavelet transform. In Research and Development, 2003. IEEE Proceedings Student Conference (pp. 19-22).

[5] Mawloud Guermoui, Mohamed Lamine Mekhalfi, Karim Ferroudji, Heart sounds analysis using wavelets responses and support vector machines, 2013 8th International Workshop on Systems, Signal Processing and their Applications (WoSSPA).

[6] Schmidt, S.E, Toft, E, Holst-Hansen, C, Graff, C,Detection of coronary artery disease with an electronic stethoscope, IEEE conference on Computers in Cardiology, 2007. Pp. 757-760

[7] Chetan Trivedy, Matthew Hall, Harold Ellis, Basic Sciences for MCEM, CRC Press, 2013.

[8] A. Cohen, Biomedical Signal Processing, Boca Raton, Florida: CRC Press, 1988.

Address for correspondence.

Pegah Derakhshan mehr

Fifth Floor, Medical Engineering Department, Shahid Sattari Highway ,University Square, Shahdia Hesarak Blvd -,Islamic Azad University, Science and Research Branch, Tehran Derakhshan.hp@gmail.com 